\title{
Ethical framework for head and neck endocrine surgery in the COVID-19 pandemic
}

\author{
Amy Y. Chen MD, MPH ${ }^{1}$ @ | Maisie Shindo MD $^{2}$ \\ ${ }^{1}$ Emory University, Atlanta, Georgia \\ ${ }^{2}$ Oregon Health Sciences University, Portland, Oregon \\ Correspondence \\ Amy Y. Chen, MD, MPH, Emory Department of Otolaryngology Head and Neck Surgery, 550 Peachtree Street, MOT 1135, Atlanta, GA 30308. \\ Email: achen@emory.edu
}

The COVID-19 pandemic has halted all elective surgeries, allowing only emergent surgeries, and in some hospitals time-sensitive urgent surgeries to proceed. "Mr X, This is $\mathrm{Dr}$ I'm calling to discuss with you your previously planned surgery." I have been having many conversations like this with my patients over the past weeks. Surgeries may be delayed or the patient and his/her family may need to make a heart wrenching decision whether to proceed with surgery in a hospital filling with COVID-19 patients, risking infection themselves, and without any visitors. Endocrine surgery falls into this valley where it is neither life threatening nor totally benign either. The American Association of Endocrine Surgeons (https://www.endocrinesurgery.org/assets/ COVID-19/AAES-Elective-Endocrine-Surgery.pdf) as well as the endocrine section of the American Head and Neck Surgery (https://www.ahns.info/wp-content/uploads/2020/ 03/Endocrine-Surgery-during-the-Covid.pdf) have put forth recommendations for thyroid and parathyroid conditions that would be considered urgent time-sensitive surgery. These include (a) high-risk thyroid cancers such as those with bulky central and lateral neck disease, concern for tracheal or esophageal involvement, or short doubling time (b) Graves' disease with thyrotoxicosis that cannot be controlled with anti-thyroid medications, (c) compressive large goiters with dyspnea or significant symptomatic vascular compression, (b) primary hyperparathyroidism with lifethreatening hypercalcemia that cannot be managed medically, and (e) endocrine disorders in pregnant patient that are dangerous to the health of the mother or fetus that cannot be controlled medically.

Certainly, there has been an international push to observe more well-differentiated thyroid cancer; however, what about those "smallish" cancers that are near the isthmus, near the trachea/esophagus, or with extracapsular extension? Despite their small size, these can become invasive to the degree that could result in the need to perform a more morbid procedure if surgery is delayed, and thus should be considered in the category of "time-sensitive surgery." What about indeterminate thyroid nodules with adverse molecular markers? If such nodules present with ultrasound findings that are concerning for local invasion, even though the cytologic diagnosis is not "malignant," such lesions should be treated as high-risk cancer, and surgery should not be delayed.

If proceeding with surgery, the surgeon has an ethical responsibility to discuss with the patient the potential risk of COVID-19 infection. We as surgeons have a responsibility to reduce risk of infection not only to the patient but the health care team who will be caring for the patient. At the minimum, COVID-19 testing should be performed preoperatively within 2 days of surgery, and the patient should be educated on the importance of self-isolation and necessary precautions.

If potential difficult airway is anticipated, communication and planning with anesthesia preprocedure is important. Despite that fact that the patient may test negative for COVID-19, the false-negative rate is not zero, and as such, precautions need to be taken to minimize exposure. A difficult airway may result in manipulation of the airway that could potentially be aerosolizing. Having the appropriate protective gear and all the necessary difficult airway equipment is essential in such a situation. If the patient needs fiber-optic laryngoscopy or tracheoscopy, nasal pledgets should be used in lieu of sprays. Surgery should certainly be postponed in COVID-19 positive patients. 
Scarcity of resources, surge planning, and public health mitigation efforts have all combined in a perfect storm to delay and in some situations, to deny treatment to head and neck surgery patients. Whereas some of our patients may afford a delay in their treatment, others do not have that luxury. It is incumbent upon us, as their clinicians, to integrate competing priorities into an acceptable plan.

Justice, or to be just/fair, must include a lens toward equity. One way to honor this ethical principle is to incorporate both clinical and nonclinical factors in risk assessment. Many papers have reported on the profound effect of sociodemographic factors on patient outcomes. An intersection between higher comorbidity burden and lower socioeconomic status can worsen disparities of who gets treatment. For example, algorithms that incorporate comorbidity are necessary so that resources can be allocated for the "greater good;" however, these guidelines risk heightening disparities and health inequity. Strategies to ameliorate these disparities include flexibility of treatment options, creative discharge planning, and thorough preoperative conditioning. Flexibility of treatment options include consideration of definitive chemoradiation, induction chemotherapy prior to surgery (to buy time), and resection with delayed reconstruction.

Beneficence, or to do good, is a guiding principle of ethics. With limited resources, the "good" of society supersedes the "good" of the individual. Hence, the cancellation of elective, nonemergent cases is instituted. So, it is the prioritization of surgical cases that are not likely to need ICU care, blood products, extended inpatient stay, and extensive ancillary laboratory/radiology testing.
However, these cancellations/delays in treatment/ changes in treatment can cause anxiety for both the clinician and his/her patient. Many of our patients have been waiting for several weeks for their treatment to start, only to have it be delayed or altered. How do we reassure the patient that the new plan is the best plan, given the restrictions that the COVID-19 pandemic places on systems? How do we, as clinicians, resolve our inner turmoil in delaying/denying/altering treatment?

Early data already demonstrate that COVID-19 is affecting vulnerable racial minorities at a higher disproportionate rate. To compound the adverse effects, this cohort has more access issues due to transportation, hourly job limitations, and lack of stable insurance. The delay in surgery may result in the patient's loss of insurance status due to loss of income and/or being furloughed. As we move into the next phase of easing up restrictions, such factors need to be taken into consideration in prioritizing whom we select for surgery.

\section{ORCID}

Amy Y. Chen (1) https://orcid.org/0000-0003-3563-6343

How to cite this article: Chen AY, Shindo M. Ethical framework for head and neck endocrine surgery in the COVID-19 pandemic. Head \& Neck. 2020;42:1418-1419. https://doi.org/10.1002/hed. 26240 